\title{
Type 1 or type 2 diabetes: has the ship already sailed?
}

\author{
AMY E MORRISON, ${ }^{1}$ SING YEE SIM, ${ }^{1}$ MARIE-FRANCE KONG, ${ }^{2}$ ROB GREGORY ${ }^{2}$
}

Key words: type 1 diabetes, type 2 diabetes, autoimmune, islet autoantibodies, C-peptide

\section{Introduction}

Type 1 diabetes is an autoimmune condition characterised by loss of pancreatic beta cells and therefore insulin production. C-peptide, secreted in equal amounts to insulin, can be used to assess beta cell function, the concentration being reflective of endogenous insulin production. ${ }^{1}$

Type 1 diabetes is often associated with other organ-specific autoimmune conditions, most commonly autoimmune thyroid disease, associated with thyroid peroxidase antibodies, and coeliac disease, associated with tissue transglutaminase antibodies. ${ }^{2}$ Islet autoantibodies to islet tyrosine phosphatase antigen-2 (IA-2), glutamate decarboxylase (GAD-65) and zinc transporter 8 (ZnT8) can be seen in patients who develop type 1 diabetes months to years prior to diagnosis. ${ }^{3}$ One of more of these antibodies is present in 80-90\% of patients with type 1 diabetes at the time of diagnosis. ${ }^{4}$

The diagnosis of type 1 diabetes and its differentiation from type 2 diabetes is essential to enable optimal therapeutic management: either insulin replacement and intensification or insulin sensitising treatments. It is additionally important in quantifying patient risk and enabling targeted risk factor modification. Diabetes is a well-known risk factor for cardiovascular disease and early mortality, which is particularly apparent in individuals with type 1 diabetes. ${ }^{5}$ Establishing a diagnosis of type 1 diabetes would additionally be associated with a known increased risk of lifethreatening diabetes-related morbidity, notably diabetic ketoacidosis $^{6}$ and severe hypoglycaemia. ${ }^{7}$

\section{Case report}

\section{Patient information}

A 37-year-old Caucasian man with a 13-year history of Addison's disease (diagnosed at age 24 years, adrenal antibodies were not measured) presented during Endocrine Clinic follow-up in 1992

Endocrinology and Diabetes SpR, University Hospitals of Leicester NHS Trust, Leicester, UK

2 Diabetes and Endocrinology Consultant, University Hospitals of Leicester NHS Trust, Leicester, UK

Address for correspondence: Dr Amy E Morrison

Endocrinology and Diabetes SpR, University Hospitals of Leicester NHS

Trust, Leicester, LE1 5WW, UK

E-mail: amymorrison15@doctors.org.uk

Br J Diabetes 2021;21:116-119

https://doi.org/10.15277/bjd.2021.297 suffering from symptomatic hyperglycaemia, with polyuria, polydipsia and weight loss $(8 \mathrm{~kg}$ ) of 3 weeks duration. He did not describe any indigestion, change in bowel habit or vomiting. At that time his regular medications were cortisone acetate $25 \mathrm{mg}$ and $12.5 \mathrm{mg}$ daily and fludrocortisone $100 \mu \mathrm{g}$ daily. He was working as a hosiery mechanic, smoked 2 cigarettes per day and drank on average 16 units of alcohol a week.

\section{Clinical findings}

On examination he was thin with muscle wasting compared with his previous clinic appointment. His weight was $71 \mathrm{~kg}$ (body mass index (BMI) $25 \mathrm{~kg} / \mathrm{m}^{2}$ ) and urine dipstick revealed 4+ glucose and $1+$ ketones with blood glucose $19.7 \mathrm{mmol} / \mathrm{L}$. Blood ketones, $\mathrm{pH}$, bicarbonate and $\mathrm{HbA}_{1 \mathrm{c}}$ were not checked at this time. He was immediately commenced on twice daily Humulin I insulin, initially 10 units twice daily. This was titrated over the next 2 months to 20 units twice daily, with resolution of symptoms and weight regain to $75 \mathrm{~kg}$.

\section{Timeline}

Since diagnosis he has continued on Humulin I insulin twice daily (Figure 1). In 2001, aged 46 years, he was found to be hypothyroid and was treated with levothyroxine; thyroid antibodies were not measured. In January 2002 his cortisone was increased to $25 \mathrm{mg} / 12.5 \mathrm{mg} / 12.5 \mathrm{mg}$, which was later converted in 2011 to hydrocortisone $10 \mathrm{mg} / 10 \mathrm{mg} / 10 \mathrm{mg}$.

\section{Diagnostic assessment}

In view of his presenting features with a history of Addison's disease, a diagnosis was made of type 1 diabetes and insulin was commenced. With a treatment regimen of twice daily isophane insulin and no requirement for soluble insulin, 22 years after diagnosis in 2014 his C-peptide level was checked and was $1417 \mathrm{pmol} / \mathrm{L}$ (eGFR 70). Subsequently, anti-GAD, insulin and islet cell antibodies were checked in 2017 and were negative (<5 IU/ $\mathrm{mL}$ ), questioning the diagnosis of type 1 diabetes in this case.

\section{Theraputic intervention}

This man has been treated with Humulin I in addition to ramipril (hypertension), rosuvastatin and ezetimibe (hyperlipidaemia), levothyroxine (hypothyroidism) and hydrocortisone/fludrocortisone (Addison's disease). His diabetes has been managed for 28 years on isophane insulin alone.

Follow-up and outcomes

The patient has overall good diabetes control (Figure 1) and a 
Figure 1. Timeline: changes in $\mathrm{HbA}_{1}$, weight and total daily dose (TDD) of insulin (1993-2019)

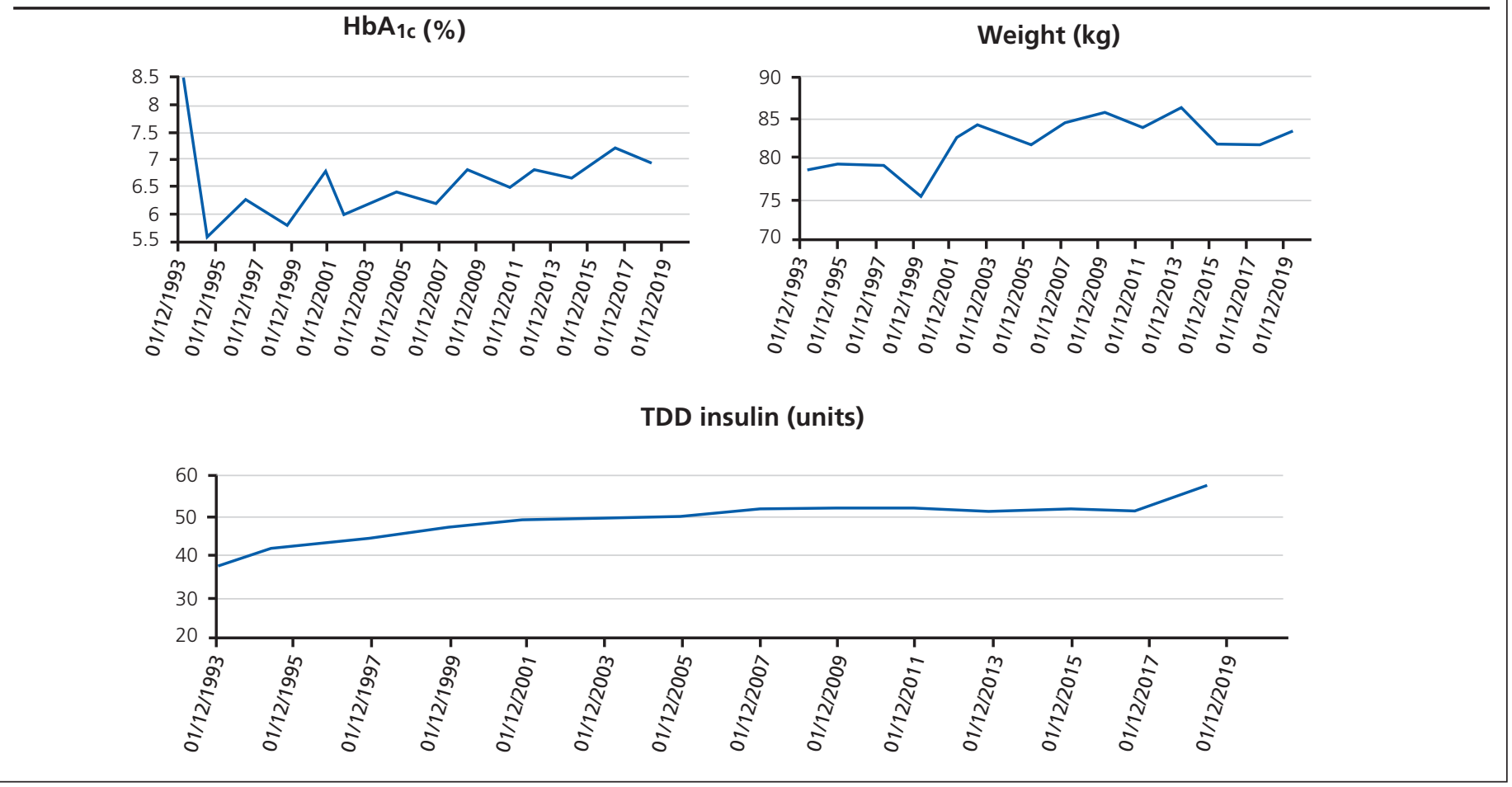

relatively stable cardio-metabolic risk profile. He continues to be followed up annually in the Diabetes outpatient clinic.

\section{Discussion}

The National Institute for Health and Care Excellence (NICE) guidelines for diagnosis of type 1 diabetes in adults suggest that type 1 diabetes should be diagnosed in adults presenting with clinical features of hyperglycaemia associated with ketosis and rapid weight loss, most commonly with symptom onset $<30$ years of age, BMI $<25 \mathrm{~kg} / \mathrm{m}^{2}$, in individuals with a personal and/or family history of autoimmune disease. It is not routinely recommended to check islet autoantibody or C-peptide levels unless there are atypical features in a patient's clinical presentation, a longer duration of symptoms of hyperglycaemia, those aged $>50$ years or with a $\mathrm{BMI}>25 \mathrm{~kg} / \mathrm{m}^{2} .{ }^{8}$ The patient discussed in this case had the diagnostic features described to make a clinical diagnosis of type 1 diabetes at presentation, and he was promptly appropriately managed with insulin therapy.

However, the Association of British Clinical Diabetologists (ABCD) Standards of Care for management of adults with type 1 diabetes suggest that age at diagnosis is the strongest indicator of diabetes type; individuals with classical clinical features at onset may in fact have type 2 diabetes. These Standards of Care state that hyperglycaemia, ketosis (without acidosis) and a personal history of other autoimmune disease are weak discriminators of diabetes type. Measurement of GAD, IA-2 and/or ZnT8 islet autoantibodies is recommended in adult onset diabetes, as in this case, to assist in clarification of diabetes type. ${ }^{9}$ Autoantibody testing is, however, recommended within 3 years of diagnosis but, in this case, was not performed until 25 years after diagnosis.
The $A B C D$ guidelines recommend that $C$-peptide is measured at least once $>3$ years after diagnosis in all patients diagnosed with type 1 diabetes. A result of $>600 \mathrm{pmol} / \mathrm{L}$ (non-fasting), as in this case, is confirmatory of a lack of absolute insulin requirement and suggests the individual may be managed with alternative therapies to insulin. ${ }^{9}$ Persistent C-peptide has been studied in a Scottish population, measuring random C-peptide in 859 individuals with type 1 diabetes of $>3$ years duration as part of routine clinic review. A C-peptide level of $>200 \mathrm{pmol} / \mathrm{L}$ was used to highlight individuals who required further diagnostic assessment relating to diabetes type. This study enabled reclassification of diabetes type in $6.8 \%$ of patients; however, insulin treatment was only stopped in $1.5 \%$ of the patients studied. ${ }^{10}$

The American Diabetes Association (ADA) standards of medical care for diagnosis and classification of diabetes type acknowledge that some individuals cannot be diagnosed with diabetes type at the time of diagnosis and that time may be required to appreciate beta cell deficiency. These standards suggest measuring islet autoantibodies in adults presenting with diabetes who do not have any traditional risk factors for type 2 diabetes such as obesity, hypertension, dyslipidaemia or a family history of type 2 diabetes, as would have been applicable for the case reported here at the time of diagnosis. ${ }^{11}$ More recently, classification of diabetes type has obvious implications in treatment options and eligibility for technology including continuous subcutaneous insulin infusion, continuous and flash glucose monitoring.

The patient in this case presented acutely with a clinical picture of symptomatic hyperglycaemia associated with weight loss and an element of ketosis (1+ urine). He was managed at this time as having a diagnosis of type 1 diabetes and commenced 
on insulin, with no trial or addition of oral glucose-lowering therapies. Due to curiosity regarding his stable glycaemic control on an atypical insulin regimen for a patient diagnosed with type 1 diabetes (Figure 1), his C-peptide levels were checked 22 years post diagnosis and were found to be at a detectable level (1417 pmol/L), indicating persistent pancreatic insulin secretory function. Throughout treatment he has remained on isophane insulin alone, maintaining an $\mathrm{HbA}_{1 c}$ of $5.5-8.5 \%$ (37-69 mmol/ mol). In people with adult age onset of type 1 diabetes, beta cell loss and therefore C-peptide level reduction is slower than in those diagnosed with type 1 diabetes in childhood, potentially associated with a lower genetic load. ${ }^{12}$ It has widely been regarded that C-peptide levels fall within 3-5 years of diagnosis; ${ }^{1}$ however, more recently, larger studies have shown variable C-peptide persistence likely correlating with the underlying genetic cause of the individual's diabetes, with as yet unknown variants. ${ }^{13} \mathrm{~A}$ study exploring the association between C-peptide levels and genetic aetiology of diabetes in 6,076 Scottish patients with clinically diagnosed type 1 diabetes or latent autoimmune diabetes of adulthood found that the majority of patients with C-peptide levels $>600$ pmol/L more than 5 years after diagnosis were negative for autoantibodies. Individuals in this category had their type 1 diabetes diagnosis re-classified as 'possible type 2 diabetes', raising this as the potential alternative diagnosis in our patient. ${ }^{13}$

The patient in this case had a diagnosed autoimmune condition (Addison's disease) and, throughout the duration of follow-up, additionally developed hypothyroidism. Despite this, his islet-specific autoantibodies - when checked 25 years after diagnosis - were negative. It may be the case that the autoantibodies had disappeared or levels had substantially diminished by the time of testing in this patient. Diabetes-specific autoantibodies are much more commonly positive at younger age of diabetes onset and less frequently with increasing age and increasing length of time from diagnosis. ${ }^{4,14}$ In this case the patient did not have autoantibodies checked at the time of diagnosis and it may have been the timing at which autoantibodies were checked which contributed to a negative result. Individuals with the presence of other autoimmune conditions are, however, reported to be more likely to have positive islet autoantibodies. ${ }^{15}$ There is also the possibility of islet autoantibodies to unknown antigens, which have not yet been tested for in this case.

This patient was at high risk for developing an autoimmune condition such as type 1 diabetes, in the context of Addison's disease. Autoantibodies are regarded as a biomarker for type 1 diabetes, and future screening via autoantibody testing may be an option in population groups felt to be more susceptible to the development of diabetes. ${ }^{16}$ More study in this field would be beneficial, especially with the prospect of immunomodulatory therapy in the prevention of type 1 diabetes.

Autoantibodies can be present for years prior to diagnosis of diabetes, and are additionally reported to be positive in 1-10\% of the general population without diabetes. ${ }^{7}$ Individuals with diabetes who have these positive autoantibodies do not necessarily require immediate insulin therapy. The decision regarding

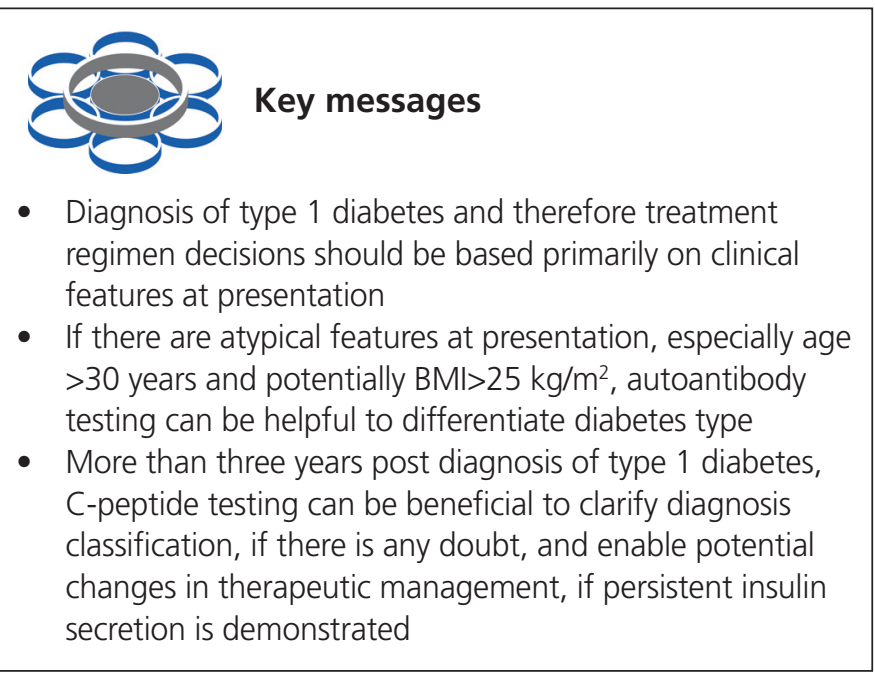

therapeutic management should be based on the clinical features of insulin insufficiency; in this case there was significant clinical concern that the patient was presenting with these, at the time of type 1 diabetes diagnosis. For this reason, management with insulin was deemed to be in the best interests of the patient for his safety at this time point. Autoantibody and C-peptide testing in this patient was carried out $>20$ years after the initial presentation and diagnosis with type 1 diabetes, and the findings of negative autoantibodies and detectable C-peptide have raised the potential need for reclassification of his diagnosis to that of 'possible type 2 diabetes'. Earlier testing of both antibodies and C-peptide in this case, nearer to the time of initial diagnosis, would have been beneficial in aiding diabetes classification and therefore guiding treatment type for this patient.

The C-peptide concentration (1417 pmol/L) confirms the patient had significant endogenous insulin reserve $>20$ years after the diagnosis of diabetes. In retrospect this should have prompted a discussion with him about the possibility of supplementing his insulin regimen with non-insulin therapies, offering the possibility of stopping insulin injections altogether.

\section{Conclusion}

Clinical features are the mainstay of the diagnosis of type 1 diabetes. Autoantibody and C-peptide levels can alter with time and can correlate with age of diabetes onset. Autoantibody testing is indicated within 3 years of diagnosis, and C-peptide testing if a longer duration of diabetes in patients with a question mark around the diagnosis of type 1 diabetes. If an individual has atypical features at presentation - notably, age $>30$ years and potentially (although it is increasingly more common) BMI $>25 \mathrm{~kg} / \mathrm{m}^{2}$ - we would suggest that autoantibody testing at diagnosis is beneficial to aid classification of diabetes type. In individuals at a greater time interval post diagnosis (>3 years), we have seen C-peptide levels to be beneficial in reclassification of diabetes type. This case highlights that, in specific patients, autoantibody and C-peptide testing is beneficial to clarify the diagnosis and to enable optimal diabetes management, with appropriate changes in treatment regimen supported by these findings. 


\section{Conflict of interest None. \\ Funding None.}

\section{References}

1. Jones AG, Hattersley AT. The clinical utility of C-peptide measurement in the care of patients with diabetes. Diabet Med 2013;30(7):803-17. https://doi.org/10.1111/dme.12159

2. Kawasaki E. Type 1 diabetes and autoimmunity. Clin Pediatr Endocrinol 2014;23(4):99-105. https://doi.org/10.1297/cpe.23.99

3. Liu J, Bian L, Ji L, et al. The heterogeneity of islet autoantibodies and the progression of beta cell failure in type 1 diabetic patients. Sci China Life Sci 2016;59(9):930-9. https://doi.org/10.1007/s11427-016-5052-3

4. Wang J, Miao D, Babu S, et al. Prevalence of autoantibody-negative diabetes is not rare at all ages and increases with older age and obesity. J Clin Endocrinol Metab 2007;92(1):88-92. https://doi.org/10.1210/ jc.2006-1494

5. Lee YB, Han K, Kim B, et al. Risk of early mortality and cardiovascular disease in type 1 diabetes: a comparison with type 2 diabetes, a nationwide study. Cardiovasc Diabetol 2019;18:157-74. https://doi.org/ 10.1186/s12933-019-0953-7

6. Vellanki P, Umpierrez G. Increasing hospitalizations for DKA: a need for prevention programs. Diabetes Care 2018;41(9):1839-41. https://doi.org/ 10.2337/dci18-0004

7. United Kingdom Prospective Diabetes Study Group (UKPDS). Intensive blood glucose control with sulphonylureas or insulin compared with conventional treatment and risk of complications in patients with type 2 diabetes (UKPDS 33). Lancet 1998;352:837-53.

8. National Institute for Health and Care Excellence (NICE). Type 1 diabetes in adults: diagnosis and management. [NG17]. 2015. Available at:
https://www.nice.org.uk/guidance/ng17/chapter/Recommendations\#diagnosis, [Accessed 30 Jan 2021]

9. Association of British Clinical Diabetologists (ABCD). Standards of care for management of adults with type 1 diabetes. Available at: https://abcd.care/sites/abcd.care/files/resources/Standards_of_Care_T1D M_ABCD_FINAL.pdf [Accessed 29 Jan 2021]

10. Foteinopoulou $E$, Clarke C, Pattenden $R$, et al. Impact of routine clinic measurement of serum $\mathrm{C}$-peptide in people with clinician diagnosis of type 1 diabetes mellitus. Diabet Med 2020 Nov 1;e14449 [online ahead of print]. https://doi.org/10.1111/dme.14449

11. American Diabetes Association. 2. Classification and diagnosis of diabetes: Standards of Medical Care in Diabetes-2021. Diabetes Care 2021;44(Suppl 1):S15-S33. https://doi.org/10.2337/dc21-S002

12. Leslie RD, Palmer J, Schloot NC, Lernmark A. Diabetes at the crossroads: relevance of disease classification to pathophysiology and treatment. Diabetologia 2016;59:13-20. https://doi.org/10.1007/s00125-015-3789-z

13. McKeigue P, Spiliopoulou A, McGurnaghan S, et al. Persistent C-peptide secretion in type 1 diabetes and its relationship to the genetic architecture of diabetes. BMC Med 2019;17(1):165. https://doi.org/10.1186/ s12916-019-1392-8

14. Fousteri $G$, Ippolito $E$, Ahmed R, Hamad A. Beta cell specific autoantibodies: are they just an indicator of type 1 diabetes? Curr Diabetes Rev 2017; 13(3):322-9. https://doi.org/10.2174/1573399812666160427104157

15. Bravis V, Kaur A, Walkey HC, et al. Relationship between islet autoantibody status and the clinical characteristics of children and adults with incident type 1 diabetes in a UK cohort. BMJ Open 2018 Apr 4;8(4):e020904. https://doi.org/10.1136/bmjopen-2017-020904

16. Eisenbarth GS. Update in type 1 diabetes. J Clin Endocrinol Metab 2007;92(7):2403-7. https://doi.org/10.1210/jc.2007-0339

\title{
$\widehat{A B C D}$ \\ Oral semaglutide (Rybelsus) Nationwide Audit Launching Soon!
}

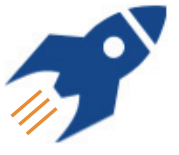

$A B C D$ is about to launch a nationwide audit of oral semaglutide in the UK to assess real-world efficacy and safety \& inform future practice and guidelines

\section{Are you using oral semaglutide (Rybelsus)?}

\author{
If yes, REGISTER YOUR CENTRE!
}

https://abcd.care/application-join-abcd-semaglutide-audit-and-gain-access-audit-tool

- you are invited to enter your patients' data into the bespoke online tool

- you will be able to analyse your local data easily

- the data will be automatically added to the national data in anonymised form

- we can provide easy-to-complete paper proformas for use in clinic if preferred

Please remember:

- the more data, the more complete our understanding of oral semaglutide in real-world practice

- all contributors will be listed in publications arising from data submission 